\title{
INTRODUCCIÓN A LA OPTOELECTRÓNICA (I)
}

\section{INTRODUCTION TO OPTOELECTRONICS (I)}

\section{AUTORES}

Arturo Pérez París: Alumno de la Escuela Politécnica. Universidad de Alcalá arturo.perez@aol.com

\section{CURRÍCULUM VITAE}

Alumno de la Escuela Politécnica de la Universidad de Alcalá de Henares (España). Ingeniero de Soporte Técnico en Kone Elevadores

\section{RESUMEN}

En este último verano (Julio - Agosto del 2.000) me dio por pensar en todos los acontecimientos acaecidos durante el curso 1.999 - 2.000 en la Universidad de Alcalá. Sus luces y sus sombras, más de éstas que de las primeras según mi criterio, aunque todo acabó bien, dentro de un orden. Ya se sabe que por las leyes de Murphy: Siempre hay algo peor y éste siempre nos alcanza en el momento menos oportuno (!). Dentro de las luces se vieron cosas asombrosas también y dignas de elogio, aunque algunos miserables no lo crean, pues ven en ellas signos de debilidad. Personalmente creo que los débiles son ellos por no ser ni capaces de reconocerlas ni dar el crédito merecido a tales personas.

\section{PALABRAS CLAVE}

Optoelectrónica - Homenots - Cesar Mataix 


\section{ABSTRACT}

In this last summer (July-August of 2000) gave me for thinking of all the events during the course 1999-2000 at the University of Alcalá. Its lights and shadows, more of them than the first in my opinion, but all ended well, within an order. Everyone knows that by the laws of Murphy: There's always something worse and it always reaches us in the wrong moment. (!) Within the lights were amazing things and also commendable, although some miserable not believe it, seeing in them signs of weakness. I personally believe that the weak are they being neither able to recognize and give due credit to such persons.

\section{KEY WORDS}

Optoelectronics - Homenots - Cesar Mataix

\section{ÍNDICE}

1. Introducción

2. Conceptos generales sobre la luz

3. Bibliografía

\section{Introducción}

En este último verano (Julio - Agosto del 2.000) me dio por pensar en todos los acontecimientos acaecidos durante el curso 1.999 - 2.000 en la Universidad de Alcalá. Sus luces y sus sombras, más de éstas que de las primeras según mi criterio, aunque 
todo acabó bien, dentro de un orden. Ya se sabe que por las leyes de Murphy: "Siempre hay algo peor y éste siempre nos alcanza en el momento menos oportuno (!)". Dentro de las luces se vieron cosas asombrosas también y dignas de elogio, aunque algunos miserables no lo crean, pues ven en ellas signos de debilidad. Personalmente creo que los débiles son ellos por no ser ni capaces de reconocerlas ni dar el crédito merecido a tales personas.

Una palabra que aprendí nueva también fue 'HOMENOTS'. Como parece, no es castellana. Es catalana y fue acuñada por el gran escritor D. Josep Plá, para definir la acción de reconocer la humanidad de alguien, sus méritos, su grandeza personal...

Con este artículo quiero rendir mi HOMENOTS personal a todas aquellas personas, profesores y alumnos, que el curso pasado con su comportamiento y saber hacer amortiguaron en lo posible los efectos negativos de la situación que todos vivimos. En particular este articulo se lo dedico especialmente a D. Cesar Mataix. Va por usted maestro.

El texto completo se dividirá en tres partes o entregas, ya que de otra forma sí que me mandarían en esta revista a "hacer gárgaras con tachuelas" por rollista. La primera será una pequeña muestra sobre 'Conceptos generales sobre la la luz'. Seguidamente pasaremos a ver de forma somera 'La teoría fotoeléctrica'. Por último, y así llegando a la parte práctica del artículo, entraremos en una exposición básica sobre los dispositivos fotoeléctricos más comunes. Comencemos pues con el tema.

\section{Conceptos generales sobre la luz}

Es sobradamente conocida la diferencia entre un ambiente iluminado y uno oscuro. Cuando nos encontramos en el primero, un conjunto de sensaciones nos permiten distinguir y reconocer los cuerpos que hay a nuestro alrededor, mientras que, en el 
segundo, dichas sensaciones de percepción del entorno dejan de producirse. Aproximadamente la mitad de todas las informaciones que recibimos los humanos son de tipo visual, es decir, que tienen como origen primario la acción de la luz. Así, en los procesos industriales, se utilizan elementos sensibles a la luz, para obtener informaciones de los trabajos realizados, de la misma manera que nuestros ojos nos lo permiten. Estos elementos sensibles a la luz reciben el nombre de fotocélulas, fotosensores o receptores fotoeléctricos. En otras ocasiones, lo que necesitamos no es captar luz sino emitirla de una determinada forma para que la reciban las fotocélulas cumpliendo unas determinadas condiciones. Estos serán los emisores. Ambos serán tratados más adelante, porque ahora la pregunta es:

¿Qué es la luz?

Como todos sabemos, o al menos intuimos, se trata de una de las manifestaciones de la energía, al igual que la electricidad, el calor, etc. La diferencia con alguna de las otras manifestaciones energéticas es que la luz no precisa de un medio material para propagarse, como lo necesitan algunas otras. Desde un punto de vista (y nunca mejor dicho) tecnológico, una de las maneras más simples de producirla es a partir de procesos de incandescencia en la que parte de la energía aplicada se transforma en calor y parte en radiación electromagnética, de ahí nuestra natural tendencia a asociar luz y calor. 


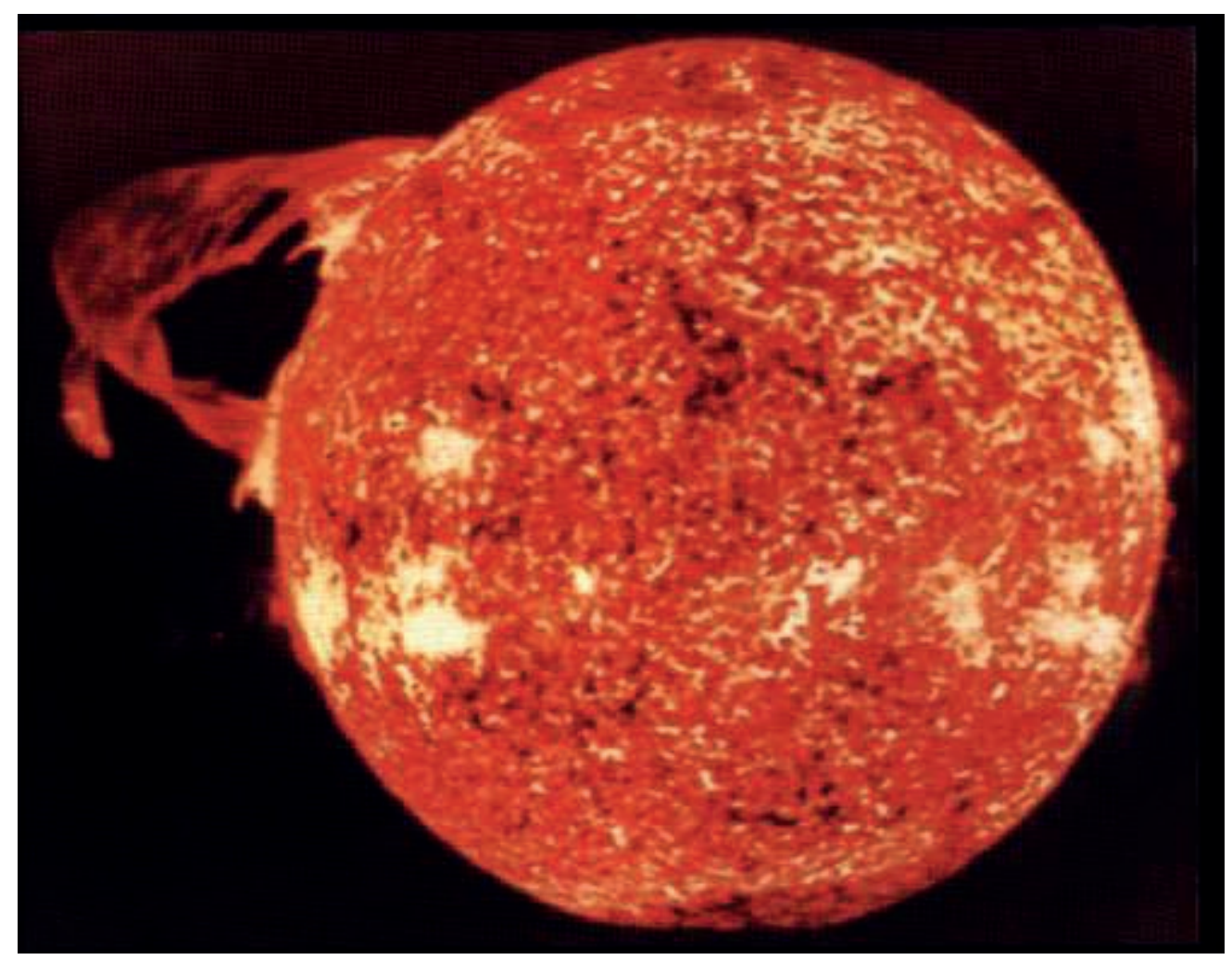

Imagen del sol en el Ultravioleta. Agosto 1973 SKYLAB

Arriba izquierda una protuberancia solar. Chorro de materia ionizada (plasma) en forma de arco, proyectado al exterior por intensos campos magnéticos

Se designa con el término general de radiación la transmisión de energía a través del espacio sin necesidad de medio material. Este transporte se efectúa por la propagación de perturbaciones periódicas del estado electromagnético del espacio. Todas las radiaciones electromagnéticas conocidas tienen en común la propiedad de propagarse en un medio a la misma velocidad; en el vacío es de $300.000 \mathrm{Km} / \mathrm{s}$ aproximadamente. Aún así existen propiedades que diferencian unas de otras, a saber:

Frecuencia: es una propiedad absolutamente invariable de la radiación y se define como el número de ciclos que efectúa la perturbación durante un segundo. Su unidad de medida es el Hertz [Hz] y se le designa la letra latina 'f' o griega 'n'. 
Amplitud: Máximo valor de la señal; en este caso de los campos eléctrico y magnético que se propagan juntos. Es una magnitud variable y que puede cambiar durante la penetración de la onda en un medio y/o a medida que el tiempo avanza en un punto cualquiera de la propagación. Se suele representar por la letra A.

Fase: Estado particular del valor de los campos, debido a que oscilan temporalmente entre dos valores máximos de signo contrario, llamados amplitud. La fase es variable, por cuanto se establece en relación al instante de tiempo para un punto dado (tiempo transcurrido desde que los campos pasaron por su máximo valor) y a la situación en el espacio, (lejanía al punto inmediatamente anterior en que la onda se encuentra en su estado de máximo valor de los campos). Se suele representar por la letra griega 'f' (fi).

Período: En vez de la frecuencia se podría tomar como una característica invariable de la radiación su inversa, el periodo; que se define como el tiempo que tardan los campos de una radiación en realizar un ciclo completo. Se expresa en segundos y la representamos por la letra ' $\mathrm{T}$ '.

Longitud de onda: es una propiedad no invariable de la misma; y se define como el camino recorrido por la radiación durante un periodo.

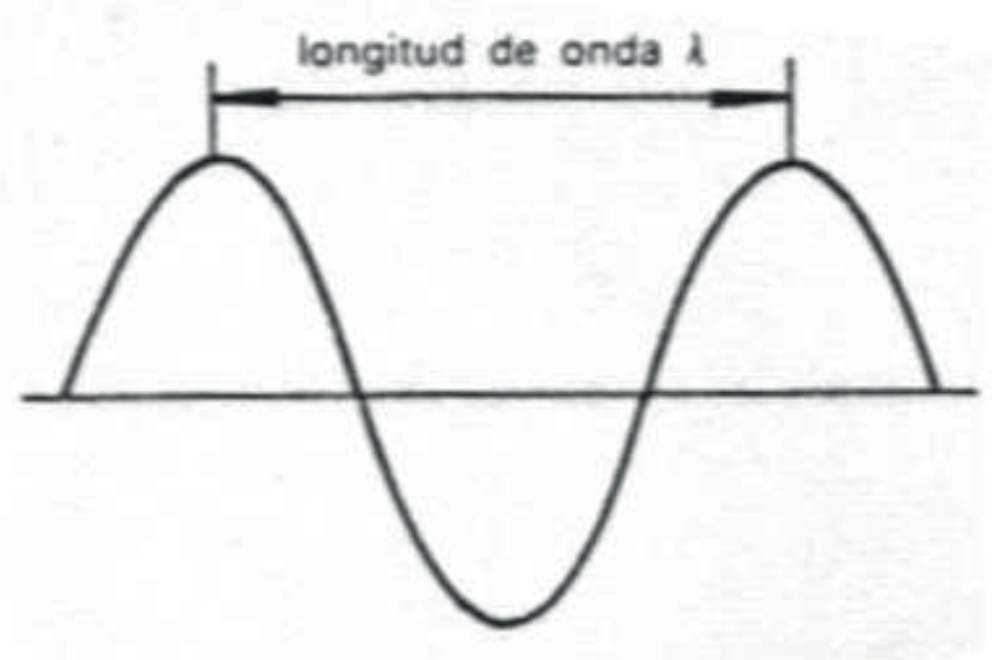


Las longitudes de onda se expresan, como es lógico, en unidades de longitud y se representan por la letra griega ' 1 ' (lambda). Hemos dicho que no es una característica invariable, puesto que la velocidad de las radiaciones electromagnéticas depende de la naturaleza del medio a través del que se propagan. Las unidades más empleadas para medir la longitud de onda de las radiaciones visibles son: el amgstrom $(\AA)$, que vale una diezmillonésima de milímetro, el nanometro ( $\mathrm{nm}$ ), cuyo valor es una millonésima de milímetro, y la micra $(\mathrm{m})$ o micrómetro que es una milésima de milímetro.

Hay un número infinito de radiaciones electromagnéticas simples, que pueden clasificarse tanto en función de la forma de ser generadas, como por sus efectos, o según sus características propias que acabamos de definir, fundamentalmente la basada en las longitudes de onda:

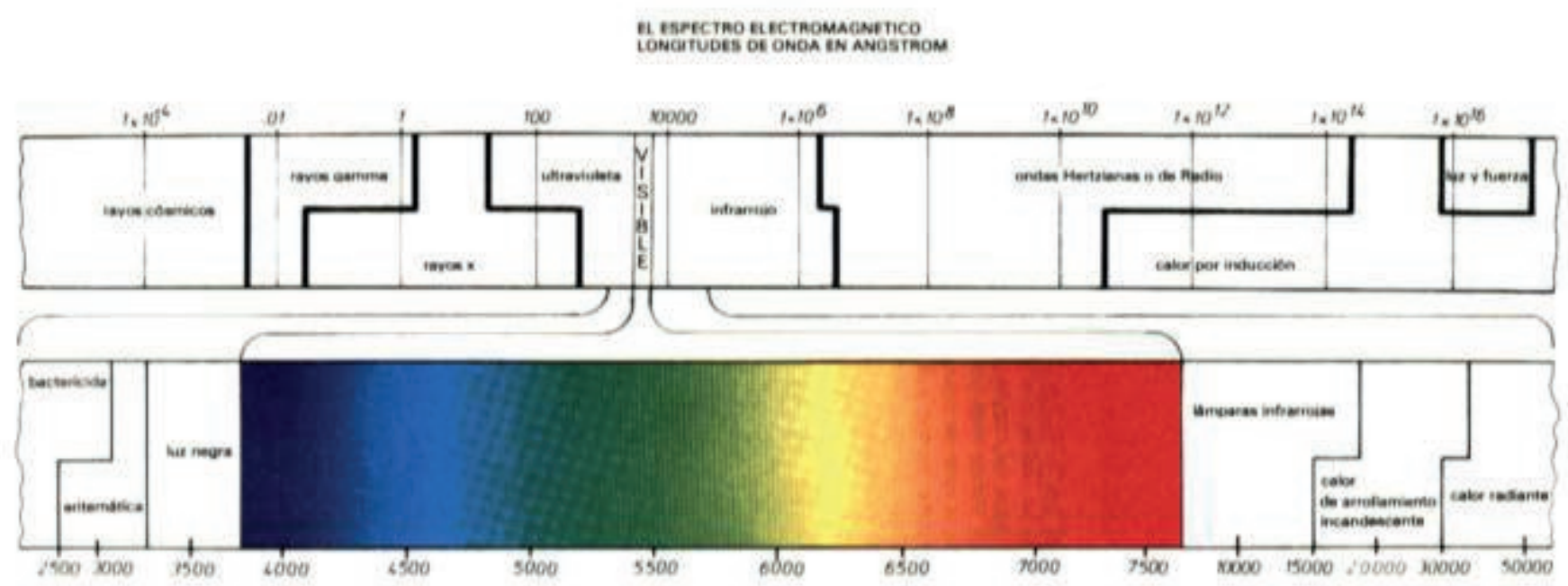



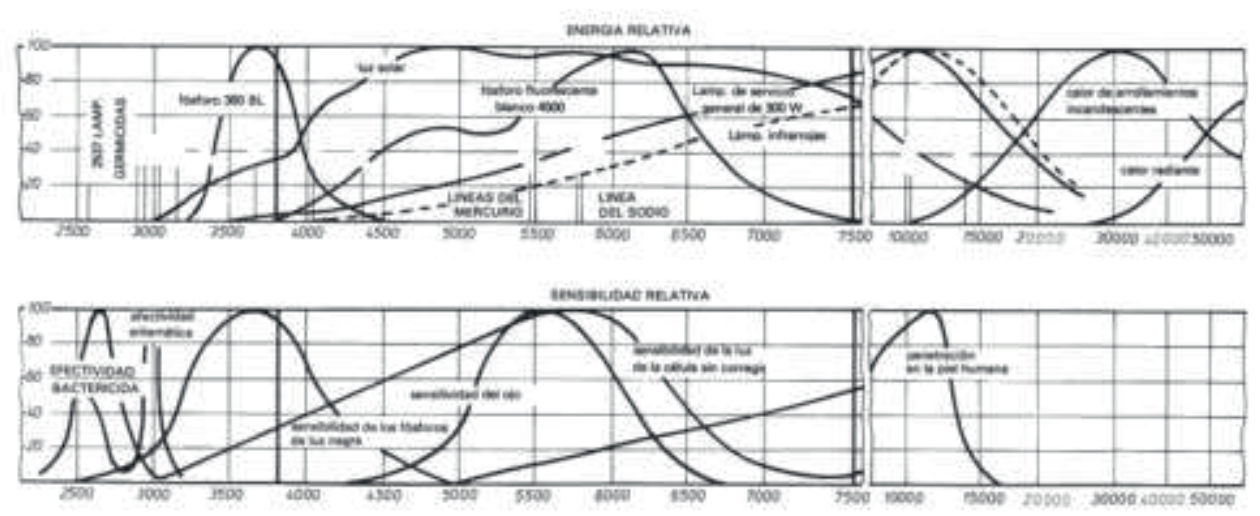

De ellas, las visibles al ojo humano ocupan una estrecha franja entre los $3.800 \AA$ y los $7.800 \AA$, limitada por las radiaciones ultravioleta (también llamada luz negra) e infrarroja que no son visibles pero son captables por dispositivos especiales y que serán los usados ampliamente en optoelectrónica. Como se observa en el anterior gráfico, resulta que el color es una de las características más importantes, por lo cual podemos concluir que las radiaciones electromagnéticas además de proporcionarnos una impresión lumínica nos producen la impresión cromática que corresponde a las diferentes longitudes de onda que percibimos.

El ojo es el órgano fisiológico que recibe la sensación de luz y es capaz de apreciar las diferencias de brillo e iluminación, siendo esto el fundamento de la visión de los cuerpos que nos rodean. Cuando nuestros ojos reciben la luz de un foco, la devuelven parcialmente en todas las direcciones, efecto que se comprueba en las fotografías con flash, si se mira al objetivo de la cámara, apareciendo nuestros ojos en rojo (como si fuéramos vampiros): 


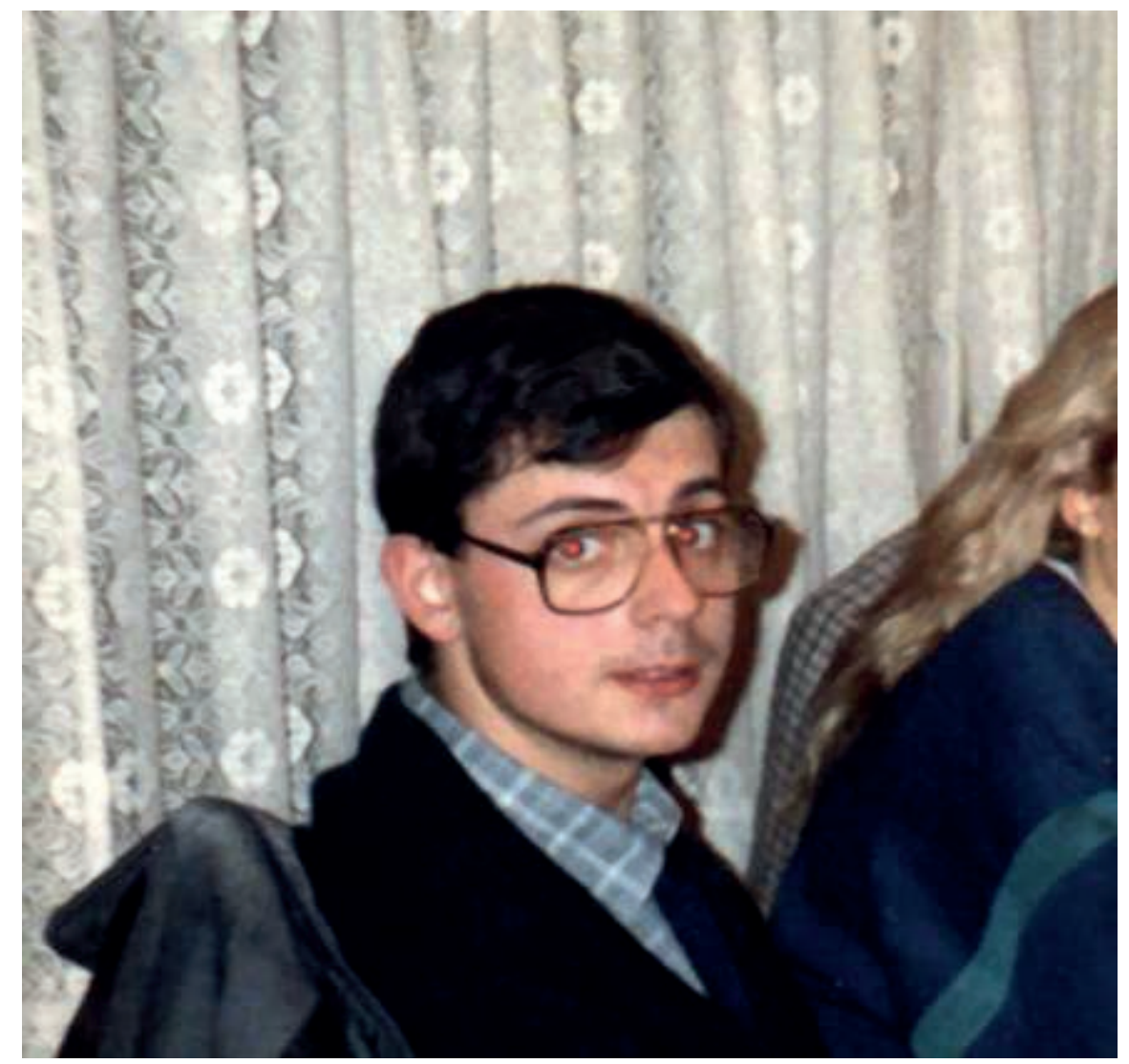

El color rojo se debe a la densa irrigación sanguínea de la retina.

Al llegar al ojo la luz que entra queda controlada "automáticamente" por un diafragma llamado iris, más o menos abierto en función de la intensidad de la onda, que es función de su amplitud. El cristalino es una lente convergente de materia elástica, que se acomoda de forma instantánea logrando que la imagen se forme con nitidez en la retina, la cual se halla provista de una serie de elementos sensibles llamados conos y bastoncillos que, a su vez, generan una serie de impulsos nerviosos que se desplazarán por el nervio óptico a la parte posterior del cerebro, donde tendrán lugar las sensaciones ópticas. 


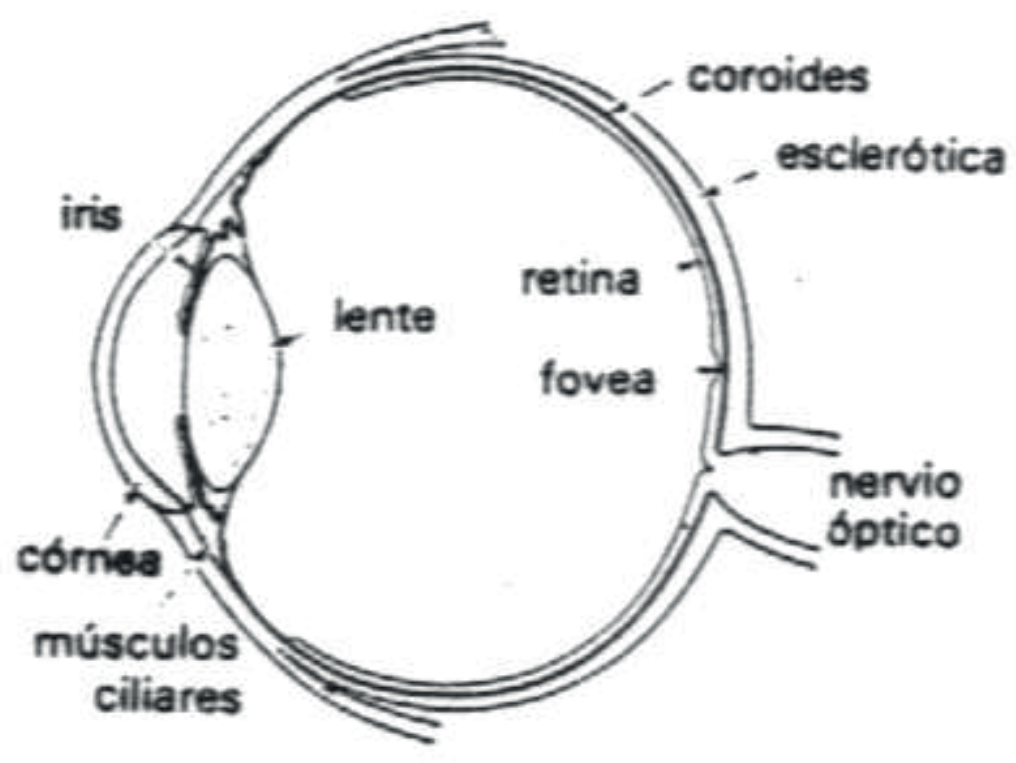

El ojo humano no tiene la misma sensibilidad de percepción para todos los colores del espectro visible, como se pude apreciar en las siguientes curvas de sensibilidad (curvas de percepción del ojo humano): 


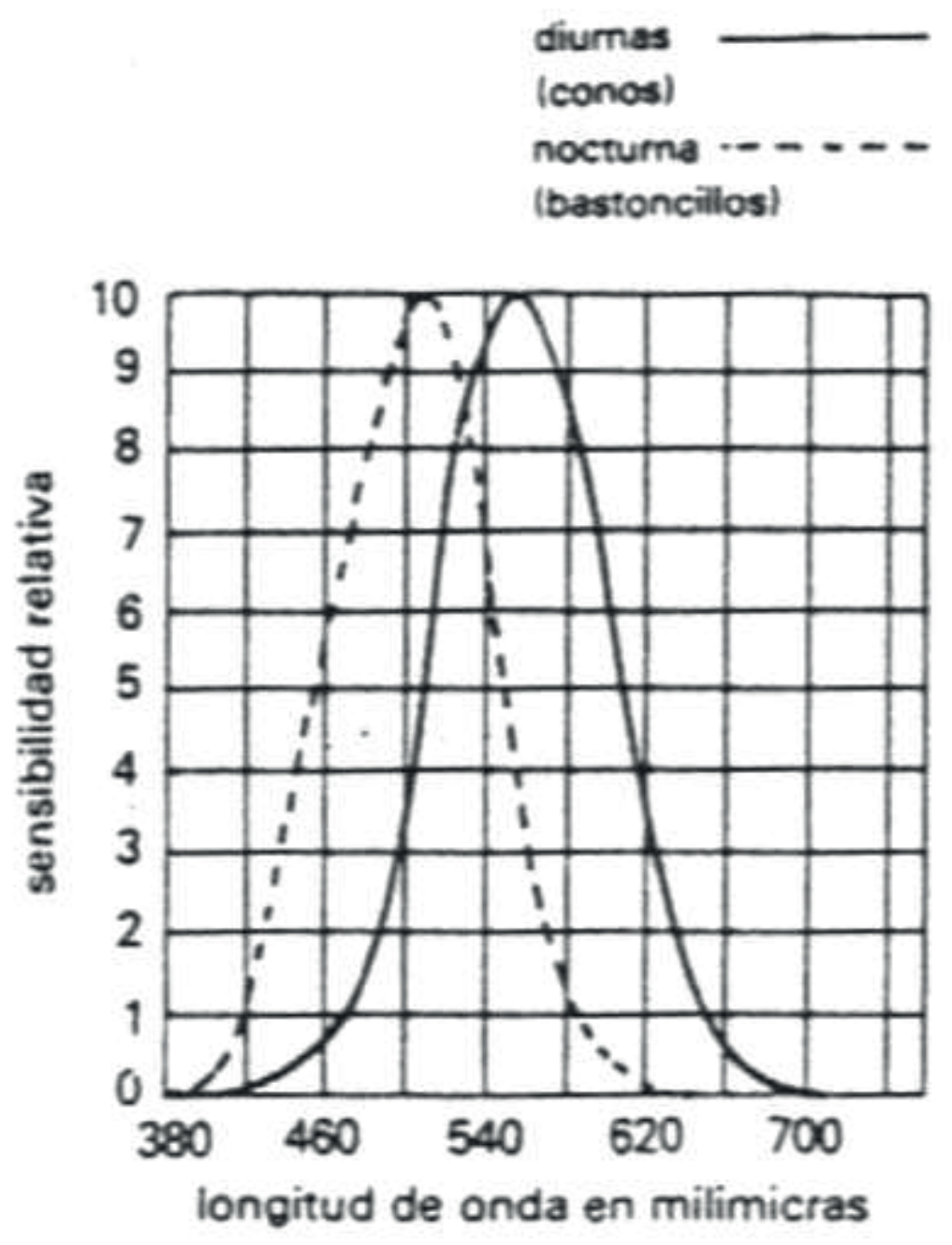

Además, como también vemos, varía con las condiciones ambientales: así para luz diurna el máximo de sensibilidad está en 555nm (amarillo - verdoso) y para la nocturna en 507nm (azul - verdoso). Realizando experiencias con gran número de sujetos, fue posible establecer, de forma gráfica, la sensibilidad espectral del ojo humano, la curva internacional de sensibilidad espectral del ojo humano: 


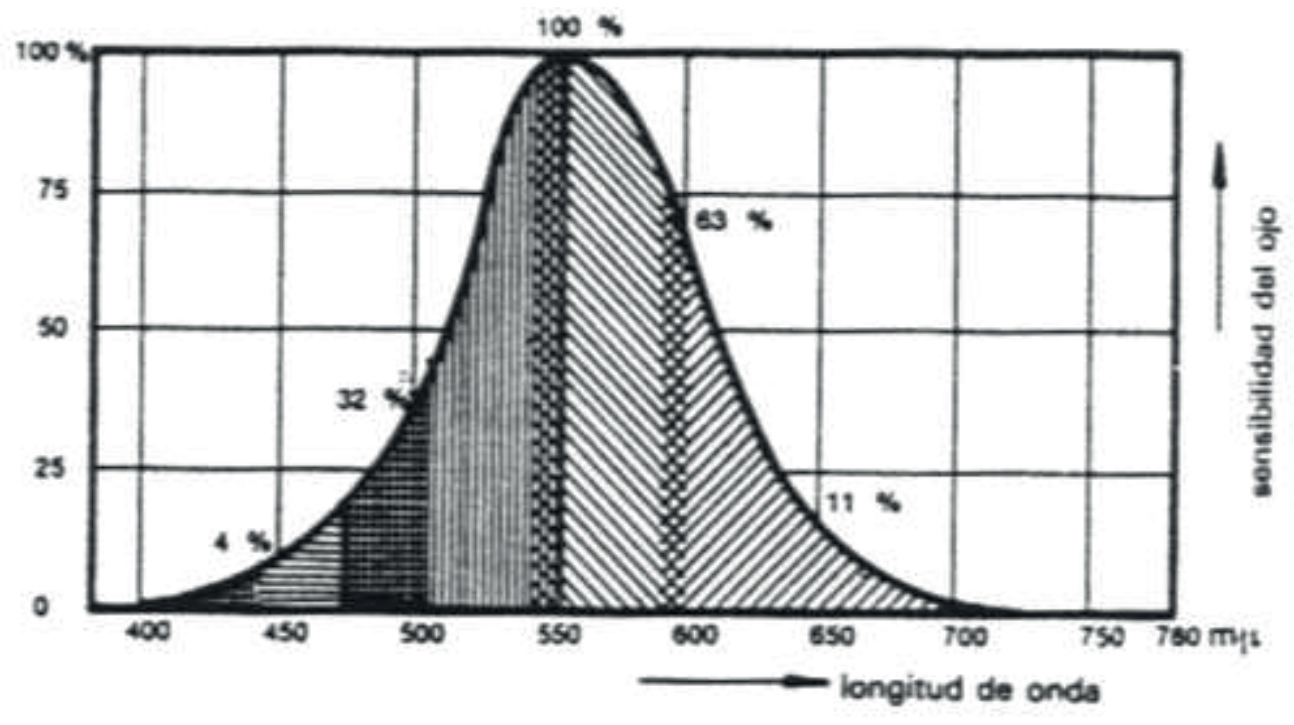

Partiendo de la base de que, para poder hablar de iluminación, es preciso contar con una fuente productora de luz y con un objeto por iluminar, las características que deben conocerse y definirse son las que se presentan a continuación:

\begin{tabular}{|l|l|c|}
\hline \multicolumn{1}{|c|}{ Magnitud } & \multicolumn{1}{|c|}{ Unidad } & Simbolo \\
\hline Flujo luminoso & Lumen $(\mathrm{Im})$ & $\$$ \\
Intensidad luminosa & Candela $(\mathrm{cd})$ & $\mathrm{I}$ \\
Nivel de iluminación (iluminancia) & Lux $(\mathrm{x})$ & $\mathrm{E}$ \\
Luminancia & Candela $/ \mathrm{m}^{2}\left(\mathrm{~cd} / \mathrm{m}^{2}\right)$ & $\mathrm{L}$ \\
\hline
\end{tabular}

La intensidad luminosa no se distribuye homogéneamente por el espacio ya que las fuentes que en la realidad se manejan no son puntuales sino con una extensión y forma definidas (de rejilla, de filamento, etc.). Por esta razón se debe introducir el concepto de ángulo sólido como una delimitación espacial de la naturaleza que a continuación se muestra: 


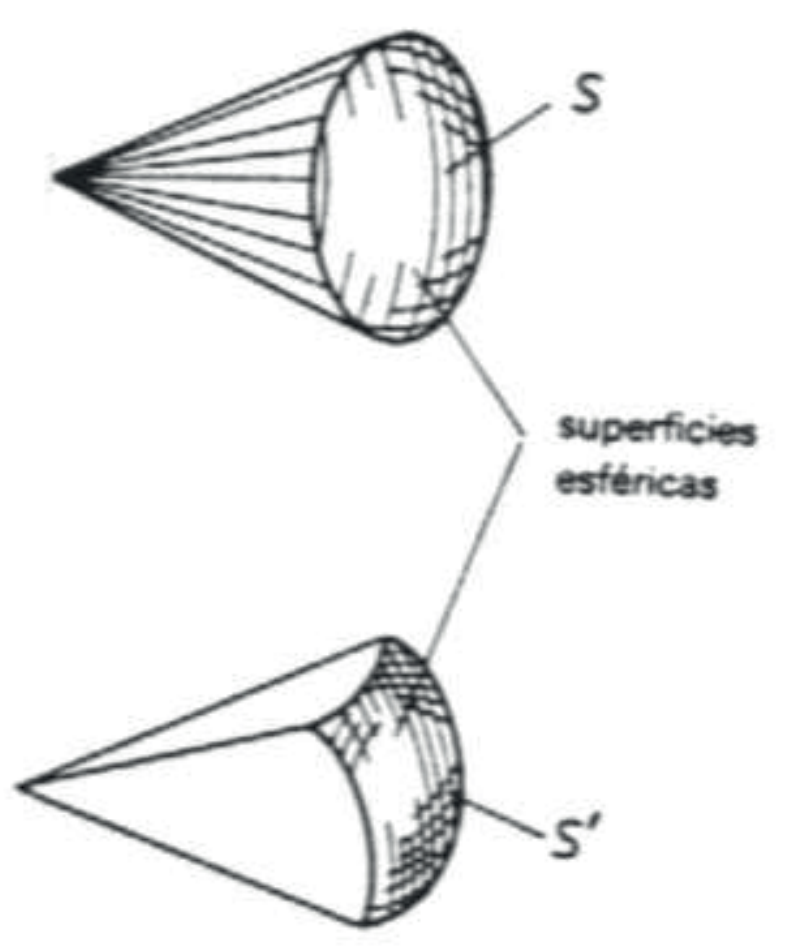

Un ángulo sólido es el espacio que se encuentra dentro de una superficie cónica o piramidal, que se obtiene al cortar con un cono o una pirámide una superficie esférica con un radio cualquiera ' $\mathrm{r}$ ' y centro en el vértice ' $\mathrm{O}$ ' del cono o la pirámide.

Los ángulos sólidos (w ) se miden en estereoradianes (sr) y se definen en general mediante la expresión: $\mathrm{w}=\mathrm{S} / \mathrm{r} 2$

La intensidad luminosa, característica fundamental de la fuente de radiación, viene dada por la energía (función de la amplitud a su vez) que emite por segundo en todas direcciones o, lo que es lo mismo, la potencia luminosa propia de la fuente que se expresa en vatios $(W)$. Sin embargo, no resulta fácil medir la potencia que corresponde exclusivamente a la región visible, ya que la mayoría de las fuentes emiten en una zona más amplia del espectro electromagnético. A efectos fotométricos sólo nos interesa la parte correspondiente al visible. La unidad del Sistema Internacional empleada, considerada básica, es la candela que se define como: Intensidad en la región visible de una fuente que emite radiación monocromática de 540 x $1012 \mathrm{~Hz}$, equivalente a 555 nm en el vacío, y cuya intensidad energética es de $1 / 683$ vatios por estereoradián.

El flujo luminoso es también una magnitud característica de las fuentes. Su unidad es el lumen (lm). 683 lúmenes equivalen a un vatio, emitidos a la longitud de onda de $555 \mathrm{~nm}$, que corresponde a la máxima sensibilidad del ojo humano. En realidad es la 
potencia (energía por unidad de tiempo) de la energía luminosa medida en relación con su efecto visual (equivale a una candela $\mathrm{x}$ estereoradián). Es decir, indica la cantidad de luz emitida por unidad de tiempo en una determinada dirección (distribución espacial de la luz emitida por la fuente).

De ello se obtiene que la intensidad luminosa de una fuente de luz en una dirección dada será la relación entre el flujo luminoso contenido en un ángulo sólido cualquiera:

$I=f / w$

El nivel de iluminación, intensidad de iluminación o iluminancia, es una magnitud propia del objeto iluminado, por cuanto indica la cantidad de luz que incide sobre una unidad de superficie de dicho objeto. La iluminancia es directamente proporcional al flujo luminoso que incide sobre una superficie e inversamente proporcional al valor de ésta:

$E=f / S$

Su unidad es el lux, equivalente a un lumen por metro cuadrado (lm m-2).

La luminancia es un concepto propio del brillo de un objeto, bien con relación a la luz de producción propia, bien reflejada (fuente que emite luz, fuente de luz sólo reflejada o fuente de luz de ambas emisiones). Se define como la sensación luminosa, que por efecto de la luz, se produce en la retina del ojo. Es la densidad superficial de la intensidad luminosa y se expresa como la relación entre la intensidad luminosa y la superficie desde la cual se emite:

$\mathrm{L}=\mathrm{I} / \mathrm{S}$ 

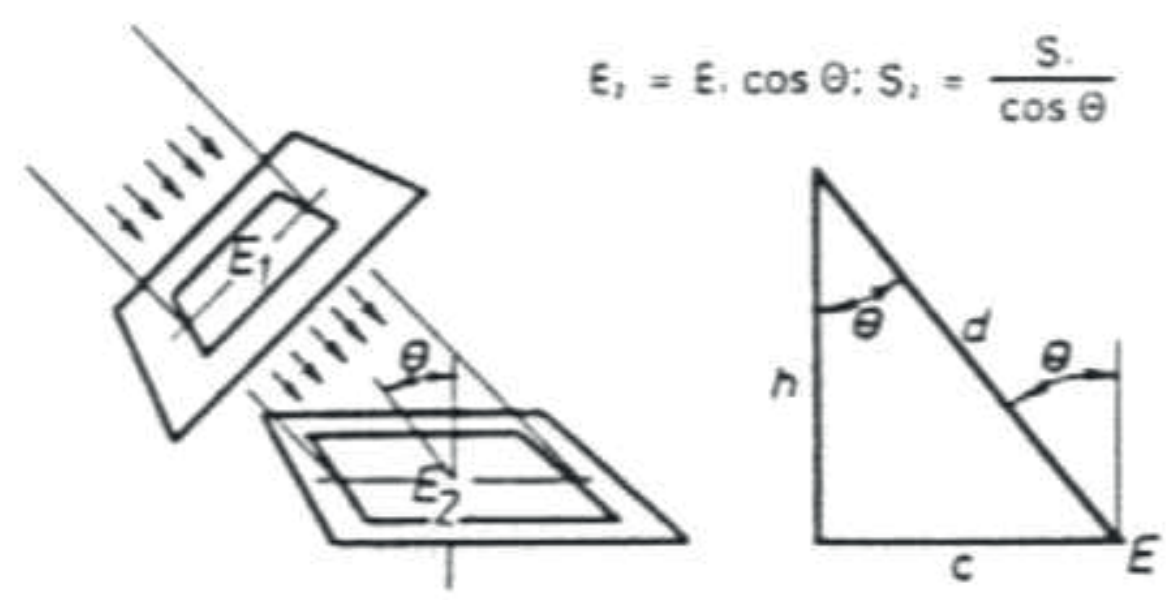

(unidad: candela por metro cuadrado, cd m-2 )

Bueno pues hasta aquí la primera entrega. Espero no haber sido excesivamente insufrible y no haberles aburrido mucho. Les espero en la próxima. Hasta entonces.

\section{Bibliografía:}

"Física", Ed. Addison Wesley Iberoamericana. Autores: Marcelo Alonso y Edward J. Finn.

"Unidades Físicas", Ed. EUDEMA. Autor: Carlos Sánchez del Río.

"Tecnología Electrónica 4", Ed. EDEBÉ. Autores: José Luis Ruiz López, Andrés Martín Blun, Carlos Lahor García, Mario Rubio García y Ricardo Casado Valero. 